\title{
The modality effect tested in children in a user-paced multimedia environment
}

\author{
M.J. Witteman*† \& E. Segers*
}

*Behavioural Science Institute, Radboud University Nijmegen, Nijmegen, The Netherlands tMax Planck Institute for Psycholinguistics, Nijmegen, The Netherlands

\begin{abstract}
The modality learning effect proposes that learning is enhanced when information is presented in both the visual and the auditory domains (e.g. pictures and spoken information) compared with presenting information solely in the visual channel (e.g. pictures and written text). Most of the evidence for this effect comes from adults in a laboratory setting. Therefore, we tested the modality effect with 80 children in the highest grade of elementary school in a naturalistic setting. In a between-subjects design, the children either saw representational pictures with speech or representational pictures with text. Retention and transfer knowledge was tested at three moments: immediately after the intervention, one day after and after one week. The present study did not find any evidence for a modality effect in children when the lesson was learner-paced. Instead, we found a reversed modality effect directly after the intervention for retention. A reversed modality effect was also found for the transfer questions one day later. This effect was robust, even when controlling for individual differences.
\end{abstract}

Keywords

long term effects, individual differences, modality effect, multimedia learning, primary school, user-paced.

\section{Introduction}

The information age is in full swing, and digital media influence more and more of our daily lives. Digital media has also found its way into classrooms, and is becoming increasingly popular. The computer provides another dimension that books cannot offer: in addition to reading information, it is also possible to listen to spoken information. Both speech and text can be accompanied by pictures. An important question is whether people learn more if information is presented in two modalities (visual and auditory; pictures and speech) rather than one (visual; pictures and text). Most research that addresses this question has focused on

Accepted: 20 September 2009

Correspondence: Mariit Witteman, Max Planck Institute for Psycholinguistics, P. O. Box 310, 6500AH Nijmegen, The Netherlands. Email: marijt.witteman@mpi.nl adults, often on college students. It is not clear, however, whether these results can be generalized to children. The goal of the present study is to test the modality effect in children and look at the influence of learner and cognitive characteristics.

\section{Literature review}

Already before the use of the computer became widespread, research had looked at the effects of combining auditory and visual stimuli (e.g. Penney 1975). An influential theory in this field is the cognitive theory of multimedia learning (Mayer 2001, 2003). This theory is based on the dual coding theory by Paivio (1986) and Baddeley's (2000) working memory model. The dual coding theory poses that there are separate channels for the processing of visual and verbal presentations and works under the assumption that the amount of 
processing that can take place within each channel is extremely limited (Paivio 1986).

Baddeley's working memory model consists of four distinct parts. Three parts, the phonological loop, visuospatial sketchpad and episodic buffer, are controlled by a fourth part, a supervisor system called the central executive. The phonological loop processes auditory information, the visuospatial sketchpad takes care of the visual and spatial information, and the episodic buffer integrates everything and adds time sequencing. The latter part is thought to have links to long-term memory.

The cognitive theory of multimedia learning (Mayer 2001) poses that there are two different channels through which information can enter the brain: a visual channel and an auditory channel. In a multimedia presentation, both channels are used. Therefore, information can be divided between two channels. Presenting all the information to one channel will only create cognitive overload, as working memory capacity is limited. Evidence for this phenomenon is also found in cognitive load theory (Paas et al. 2004). If there is cognitive overload, the brain cannot process all the incoming information, resulting in inferior learning performance. According to the modality effect, it is possible to both retain more information (retention benefits) and foster deeper learning (transfer benefits) when information is presented in two channels. This has been tested with animations or pictures (visual domain) accompanied by either text (also in the visual domain) or narration (auditory domain). The effect is only present, however, when the two streams of information are complementary: presenting additional information that is redundant, will not aid learning (Mayer et al. 2001).

\section{The modality effect tested with adults}

The existence of the modality effect in computerlearning environments has been tested on several occasions. Mayer and Moreno (1998), for example, conducted two experiments with college students. The participants either saw an animation on a computer screen accompanied by auditory information (ANcondition), or an animation accompanied by text (ATcondition). Experiment 1 focused on meteorological phenomena, while experiment 2 focused on car mechanics. The two experiments yielded the same basic conclusions: on both retention and transfer questions, the participants in the AN-condition outperformed their peers in the AT-condition. The effect sizes indicated that these effects were substantial. Therefore, the authors found evidence for the existence of the modality effect.

Another study that tested the modality effect was conducted by Brunken et al. (2004). In two withinsubjects studies, they tested two groups of 10 female students. The research focused on the influence of induced cognitive load on the modality effect. The participants completed a pre-test and then participated in an intervention in which they learned about the human circulatory system or about Florence. There were two conditions: visual (pictures and on-screen text) and audiovisual (pictures and speech) Afterwards, their knowledge was tested again. The participants in the visual condition got significantly lower scores than the participants in the audio-visual condition: a modality effect. This held for the primary (learning) task and the secondary (distracting) task.

Other studies, however, have found conflicting results with regard to the modality effect (e.g. Dean et al. 1988; Mann et al. 2002). Tabbers et al. (2004) tested 111 second year university students in a classroom setting. The participants saw diagrams on a computer with either auditory or visual instructions. Afterwards, the participants completed retention and transfer tests. The results showed a reversed modality effect, as the participants scored better on retention tests when the diagrams were paired with visual cues. In the auditory condition, the participants got lower scores on both retention and transfer tests.

An important distinction between this study and those mentioned previously can be found in the pacing of the information. Whereas the former was systempaced, Tabbers et al. (2004) allowed their participants to process the information at their own speed (learnerpaced). The authors offered this distinction as an explanation for the reversed modality effect found in their study, suggesting that when learners can decide when to start the spoken information, it is possible to process the pictorial information and the spoken information separately Therefore, the advantage of presenting information in two channels disappears. Moreover, the authors added that it was easier for the participants in the visual condition to switch between the picture and the on-screen text because these were both present at the screen throughout the intervention. For the audio files, however, it was impossible to switch back and forth between the spoken information and the pictures on the 
screen because the spoken information disappears immediately after it is presented.

\section{The modality effect tested with children}

Less is known about the modality effect in children. Only a few studies focused on children in primary school, even though this group's main focus during the day is studying. By extending the research to children, it is possible to learn more about the developmental process of the modality effect. Moreover, it is possible to study the modality effect in a more heterogeneous group as not all children in elementary school go on to a university, the population most often studied in research of the modality effect. Another important reason to study children is that they have an underdeveloped phonological loop, and therefore, working memory might work differently in adults and children (Baddeley et al. 1998). Mann (2008) incorporated this notion in the attentional control theory of multimedia learning, in which he proposes that an underdeveloped phonological loop can result in insufficient mental articulation during listening and reading. Children, therefore, might benefit more from multimedia learning when this message is aided with hints and important visual events to shift the learner's attention.

A study investigating a younger population was conducted by Mann et al. (2002). Forty-two 12-year-old participants were shown animations on a computer screen, accompanied by either visual or auditory information. Questions were presented on the screen, and the children wrote down the answers in their workbooks. This study found no differences between the experimental conditions. Similar to Tabbers et al. (2004), this study was user-paced. Mann et al. (2002) hypothesized that because the working memory processes in these children are not fully developed, there are fewer possibilities for referential connections between the auditory and visual channel. The authors did not take individual differences into account. Another explanation could be the preferred mode of presentation of the participant: Rickheit et al. (1987) found that the participants used to listening to information recalled more when tested orally, and the participants who were used to reading information performed better on written tasks.

To get more insight into the modality effect, Ginns (2005) performed a meta-analysis on 43 independent studies. Overall, Ginns found sufficient support for the modality effect, also in children (e.g. Mousavi et al. 1995; Jeung et al. 1997). This effect was larger for studies that used high interactivity elements (with interactivity defined as 'the extent to which the learning task requires the student to hold several related chunks of to-be-learned information in working memory simultaneously in order to comprehend then learn the concept or procedure', p. 320), and for system-paced studies (compared with user-paced). Age did not have a significant effect. The modality effect was largest for students that learned with virtual reality, followed by participants in computer-based studies. The studies that used audio tapes found the smallest effects.

\section{Individual differences in the effect of modality}

As indicated by Mann et al. (2002), individual differences, such as working memory span, can influence the modality effect as well. Mayer (1997) found that modality effects are strongest for participants with low prior knowledge and high spatial ability. Kozhevnikov et al. (2002) wanted to test the effects of spatial and verbal ability on people with a preference for visual learning (visualizers) and people with a preference for verbal learning (verbalizers). Within these groups, they checked the level of spatial ability. Though the verbalizers were a relatively homogeneous group, the visualizers were not. They formed two distinct groups: one with low and one with high spatial ability. High-spatial visualizers saw graphs as abstract representations, whereas low-spatial visualizers interpreted them as pictures.

Mayer and Massa (2003) wanted to find out in what way visual and verbal learners differed from one another. They discovered that there were four separate factors that, together, accounted for almost half of the variance in the visualizer-verbalizer distinction. These four factors were: cognitive style (the way a person approaches learning activities), learning preference (i.e. visual preference), spatial ability (high or low) and general achievement (as measured by mathematical and verbal scholastic aptitude test scores plus a vocabulary test). The authors concluded that studies that focused on the modality effect should aim to control for these factors.

\section{Long-term effects of modality}

An important feature of learning is remembering knowledge over time. However, the long-term effects of 
the modality effect have scarcely been studied. All studies mentioned earlier tested participants immediately after learning the materials but ignored the way presentation mode affects the consolidation of knowledge. One study that did try to shed more light on this topic was conducted by Segers et al. (2008). In a withinsubject design, 128 elementary school children were taught four different lessons in a school setting. These computerized lessons either contained solely written presentations, a written presentation accompanied by pictures, solely oral presentations, or an oral presentation accompanied by pictures. All lessons were user-paced. Segers et al. found a modality effect immediately after the learning phase for both transfer and retention tests. One week later, however, there was no significant difference between conditions for retention. For the transfer questions, a reversed modality effect was found. These results did not change when controlling for spatial ability or verbal ability. However, on the retention questions students with high verbal ability scored higher on the written conditions, but only immediately after the learning phase.

Children with high interest/prior knowledge scored higher in the oral conditions than in the written conditions on both retention tests. For the transfer condition, no effects were found initially, but after a week the results for the text-only condition were better for children with high interest/prior knowledge. These children significantly outperformed their peers in the oral conditions and there was a trend towards better performance in the written condition with pictures.

\section{Present study}

The goal of the present study was to replicate the modality effect in young children and see how this effect holds over a longer period of time. In doing so, we wanted to shed more light on the findings with adults (e.g. Mayer $\&$ Moreno 1998; Mayer 2001) and findings with children (e.g. Segers et al. 2008). We used computerized materials drafted from work by Mayer on the formation of lightning (e.g. 2001) to test the modality effect with 80 children in the highest grade of elementary school (i.e. sixth grade in The Netherlands).

The population used for the present study was much more heterogeneous than the college students used by most studies on the modality effect. Therefore, it is important to control for individual differences. Based on the previous review of the literature, the following characteristics were taken into account in the present study: preferred mode of presentation (Rickheit et al. 1987; Mayer \& Massa 2003), learning eagerness (interest, Segers et al. 2008), prior knowledge (Mayer 1997), visual working memory and auditory working memory (Mann et al. 2002), spatial ability (Kozhevnikov et al. 2002; Mayer \& Massa 2003), technical reading and reading comprehension (verbal ability, Kozhevnikov et al. 2002), and general school performance (general ability, Mayer \& Massa 2003). Our first hypothesis was that children who score higher on learning and cognitive measures also score higher overall on the retention and transfer questions.

Similar to Segers et al. (2008), we wanted to study the long-term effects of the modality effect.

Because Segers et al. (2008) found different effects after one week, the current study added another measuring moment to gain more information about the processes involved in learning from different modalities. We had three different testing occasions, one immediately after the intervention, a day after the intervention, and a final test a week after the intervention. During every testing phase, the children were presented with both retention and transfer questions.

Our second hypothesis was to find a modality effect immediately after the intervention for retention, which would disappear at the second testing occasion and remain gone at the third testing occasion, thereby replicating and extending the results found by Segers et al. (2008). Our third hypothesis was to find the same pattern for the transfer questions. We expected to find this because there are indications that knowledge consolidates after one night (McLelland et al. 1995), resulting in a long-term effect that does not differ from the effect expected after one week. In addition, the fourth hypothesis was that modality effects for both retention and transfer would interact with prior knowledge, spatial ability (Mayer 1997) and preferred mode of presentation (Rickheit et al. 1987).

\section{Method}

\section{Participants}

The participants were 80 children ( 35 girls, 45 boys) in sixth (senior) grade of a public elementary school in a suburban area near Rotterdam, the second largest city of 
The Netherlands. Their ages ranged from 10.8 to 13.3 years old, with a mean age of 11.8 years $(\mathrm{SD}=0.5$ ).

The experiment took place during class time. All parents were informed of the study and had the possibility to withdraw their children from the study. In exchange for participating with the study, the school received a fee of 160 euros. Ninety percent of the participants were born in The Netherlands, and $76 \%$ had parents who were also born in The Netherlands. Of all the participants, $82.5 \%$ stated that the only language spoken at home was Dutch. Ten children (12.6\%) attested that they spoke both Dutch and another language at home, where for half of these, the second language was a Western language. Only two participants spoke no Dutch in their household, but rather spoke a non-Western language.

The participants were randomly assigned to a condition (auditory or visual) and the order of subsequent tests. Because of the absence at some of the test days, a few of the children did not perform all the tests, resulting in slightly different degrees of freedom scores in the results section. In the final analyses, 14 children with a preference for reading were in the reading condition and 13 in the listening condition. Of the children with a preference for listening, 20 were in the reading condition and 24 in the listening condition.

\section{Materials}

\section{Child characteristics}

In addition to the experiment, a number of tests were completed by the participants. These tests could be divided into two main categories: learning characteristics and cognitive characteristics. All of the tests were administered by the researcher or a certified teacher before the learning intervention.

\section{Learning characteristics}

Before the learning intervention took place, the participants were asked to rate their preferred mode of presentation (reading or listening), learning eagerness on the topic of meteorology and prior knowledge. All were assessed with a single forced-choice question (the latter two variables on a five-point scale).

\section{Cognitive characteristics}

Visual working memory was tested using the visual working memory test of the Nederlandse Differentiatie Testserie [Netherlands Differentiation Test Series] 2001 (van Hoorn et al. 2003). This task was composed of three identical parts, including a training session. In every part, the participants studied a page with 10 drawings for one minute. Next, another page with 10 drawings was presented for an additional minute. After that, the participants were required to write down all the items they remembered.

Auditory working memory was tested with a digit span test. This test was part of the Wechsler Intelligence Scale for Children-III for Children (Wechsler 1992) and was composed of two parts. In the first part, the participants had to repeat a sequence of digits that started with two digits and ended with eight. They received two attempts for every number of digits. The test was over if both attempts were incorrect. In the second part, the participants were required to repeat the digits in reverse order. Again, if both attempts failed, the test was stopped.

A mental rotation task (van Boxtel et al. 1980) was used to assess spatial ability. This test was composed of 15 exemplar figures that were either rotated or mirrored in five different ways. The instruction was to indicate which figures were rotated. After presenting these instructions and answering all questions, the children had five minutes to complete as many items as possible. Both their degree of completion and the number of correct answers were recorded.

As part of their regular school programme, the participants were tested for their technical reading ability using the last card of the Drie Minuten Toets [Three Minutes Test] (Verhoeven 1995). This card consists of 120 words that have at least two and at most four syllables. The students were required to read out loud as many words as possible during one minute.

All of the children also participated in the Cito (Centraal instituut voor toetsontwikkeling) test 2007. Every year, this standardized test is taken by approximately 154000 children in the highest grade of primary school in The Netherlands (van der Lubbe 2008) and helps decide which level of secondary education is appropriate for a student. The test consists of many different parts, but only the score for general school performance and reading comprehension were used for this study.

\section{Materials for the intervention}

The materials used for this study were adapted from the work of Mayer (e.g. Mayer 2001). We used the material on the formation of lightning. For the purpose of this 
study, the materials were translated by the first author, in cooperation with a professional translator. Because the original material was intended for use with adults, the translations used were slightly simplified where necessary. The content was not changed. Two certified teachers judged the materials suitable for the children.

\section{Test materials}

Three test versions were created to assess knowledge. The order in which the tests were taken was randomized. The participants were tested immediately after, a day after and one week after the intervention. All of the tests contained one identical retention question, a translation of the original retention question in Mayer (2001). Every test also contained two open-ended transfer questions. Four of these questions were translated versions of Mayer's (2001) transfer questions, the other two created by the researchers. All questions can be found in Appendix A.

Scoring was performed by the first researcher. Points were assigned if the elements mentioned in the answer model appeared. The second researcher checked $10 \%$ of the sample (assigned randomly across time points and conditions). The inter-rater reliability was high, $r=0.92, P<0.01$.

\section{Procedure}

The experiment was run on the computers available in the school. At each given moment, 7-9 students were participating in the experiment in the auditorium of the school. The experiment leader was present for the testing.

The program used for this study was Microsoft PowerPoint. The students were assigned randomly to a condition (visual or auditory) as they presented for the experiment. Before starting the experiment, the students were asked for their names, preferred mode of presentation, learning eagerness and prior knowledge (see learning characteristics). In the visual condition, the participants were shown 16 pictures with one or two lines of text placed directly underneath. Every picture with the accompanying stimulus material was presented on a separate slide.

For the auditory condition, a link to the sound file was presented at the same place as the text of the visual condition. The children clicked to start the audio presentation. The sound file contained the same information as the slides in the visual condition, and was recorded using the voice of the experimenter, who was raised in the regional area of the school. The computers for the auditory condition were connected to headphones. The participants were able to freely browse between the 16 slides and read or listen to the learning material as often as they felt necessary.

Immediately after the intervention, the children were presented with the first test. Afterwards, the computer program gave the instruction to the students to return to their teacher. There was no time limit for any part of this experiment.

\section{Analyses}

To test the first hypothesis, we calculated correlations between learning and cognitive characteristics, as well as the scores on the different retention and transfer tests.

To test the second and third hypotheses, general linear model (GLM) repeated measures analyses were used with time (immediately after intervention, one day after intervention, and one week after intervention) as a within-subject factor, and condition (reading, listening) and preferred mode of presentation (reading, listening) as between-subjects factors. The following centred covariates were entered: learning eagerness, prior knowledge, visual working memory, auditory working memory, spatial ability, technical reading ability and reading comprehension. The alpha level for all the tests was 0.05 .

To test the fourth hypothesis, the same GLM analysis was conducted, additionally testing for the interaction between condition and the following: prior knowledge, spatial ability and preferred mode of presentation.

\section{Results}

Table 1 shows the scores of the participants at the different testing moments on both the reading and the listening conditions and for both retention and transfer questions. Table 2 shows the mean scores and SDs for the learner and cognitive characteristics.

\section{Correlations between cognitive and learning characteristics and test scores}

Correlations between the different learning and cognitive characteristics and these test scores were calculated. General school performance correlated very 
Table 1. Mean scores and standard deviations for retention and transfer questions.

\begin{tabular}{llllll}
\hline & \multicolumn{2}{c}{ Retention } & & \multicolumn{2}{c}{ Transfer } \\
\cline { 5 - 6 } & \multicolumn{2}{c}{ Mean (SD) } & & \multicolumn{2}{c}{ Mean (SD) } \\
\cline { 5 - 6 } & Listening & Reading & & Listening & Reading \\
\hline T1 & $4.95(2.40)$ & $5.60(3.18)$ & & $2.18(1.06)$ & $1.85(1.12)$ \\
T2 & $4.46(2.02)$ & $4.18(2.46)$ & & $1.92(1.16)$ & $2.36(1.08)$ \\
T3 & $3.73(2.04)$ & $3.58(2.25)$ & & $1.73(1.06)$ & $1.82(0.98)$ \\
\hline & & & & \\
\hline
\end{tabular}

Table 2. Mean scores and standard deviations (SDS) for learner and cognitive characteristics

\begin{tabular}{|c|c|c|c|}
\hline & Mean & SD & $\begin{array}{l}\text { Maximum } \\
\text { score }\end{array}$ \\
\hline \multicolumn{4}{|l|}{ Learner characteristics } \\
\hline Previous knowledge & 2.59 & 0.79 & 5 \\
\hline Learning eagerness & 3.27 & 0.94 & 5 \\
\hline \multicolumn{4}{|l|}{ Cognitive characteristics } \\
\hline Technical reading ability & 93.00 & 17.12 & 150 \\
\hline Deep reading ability & 60.78 & 17.85 & 120 \\
\hline Spatial memory & 46.48 & 11.36 & 100 \\
\hline Auditory working memory & 13.68 & 2.61 & 20 \\
\hline Visual working memory & 32.73 & 4.51 & 40 \\
\hline $\begin{array}{l}\text { General school } \\
\text { performance }\end{array}$ & 536.5 & 9.60 & 550 \\
\hline
\end{tabular}

highly with reading comprehension ( $r=0.851$, $P<0.001)$ and was therefore left out of subsequent analyses. Prior knowledge, $(r=0.272, P=0.015)$, auditory working memory, $(r=0.503, P<0.001)$ and visual working memory $(r=0.385, P<0.385)$ also correlated with reading comprehension, but as $r$ was never higher than 0.6 , these were kept in the analyses.

For the retention questions, significant positive correlations were found for prior knowledge at $\mathrm{T} 1$ $(r=0.268, P=0.016)$, visual working memory at $\mathrm{T} 1$ ( $r=0.247, P=0.028$ ), auditory working memory at T1 $(r=0.509, P<0.001), \mathrm{T} 2(r=0.478, P<0.001)$, and T3 $(r=0.338, P=0.003)$ and reading comprehension at T1, T2 and T3 $(r=0.540, r=0.524, r=0.472$, respectively, $P<0.001$ ), For the transfer questions, significant positive correlations were found for prior knowledge at T1 $(r=0.252, P=0.024)$ and reading comprehension at T2 $(r=0.324, P=0.004)$ and T3 $(r=0.338, P=0.003)$.

\section{Retention questions}

The GLM repeated measures analysis showed a main effect of time, $F(2,60)=22.940, P<0.001$, partial $\eta^{2}=0.443$ and an interaction effect for time and condition, $F(2,60)=4.197, P=0.020$, partial $\eta^{2}=0.123$. The children who scored high on reading comprehension had higher scores overall, $F(1,61)=17.576$, $P<0.001$, and there was a similar trend for learning eagerness, $\quad F(1, \quad 61)=3.829, \quad P=0.055, \quad$ partial $\eta^{2}=0.059$.

Within-subjects contrasts showed that the interaction effect between time and condition was significant at T1 vs. T2 $(P=0.006)$ but not at T2 vs. T3 $(P=0.977)$. An ANCOVA with the same variables, only looking at T1 and $\mathrm{T} 2$, revealed that the scores in the reading condition were higher than the scores in the listening condition at T1 $(B=1.162, P=0.035)$ but not at T2 $(B=-0.30$, $P=0.946)$. Reading comprehension remained significant at both measurements $(P<0.001, P=0.003)$. We thus found a reversed modality effect for retention at $\mathrm{T} 1$, which was not present anymore at T2 and T3. Figure 1 depicts these results.

\section{Transfer questions}

The GLM repeated measures analysis showed a main effect of time, $F(2,60)=4.505, P=0.015$, partial $\eta^{2}=0.133$, and no interaction effects. Furthermore, there was a main effect of reading comprehension, $F(1$, $61)=10.570, P=0.003$, partial $\eta^{2}=0.148$, indicating that the children who scored high on reading comprehension had higher scores overall, and a similar effect for prior knowledge, $F(1,61)=3.996, P=0.050$, partial $\eta^{2}=0.061$.

The within-subjects contrasts showed that the interaction effect between time and condition was significant at T1 vs. T2 $(P=0.042)$ but not at T2 vs. T3 $(P=0.244)$. An ANCOVA with the same variables, only looking at $\mathrm{T} 1$ and $\mathrm{T} 2$, revealed that the scores in the reading condition were higher than the scores in the listening condition at T2 $(B=0.614, P=0.024)$ but not at T1 $(B=-0.208, P=0.451$.). Reading comprehension again showed a main effect, $F(1,61)=6.211$, $P=0.015$, partial $\eta^{2}=0.092$. We thus found a reversed modality effect for transfer at $\mathrm{T} 2$ and therefore partly replicated Segers etal. (2008). These results are depicted in Fig 2. 
Fig 1 Scores on retention questions over time.

Fig 2 Scores on transfer questions over time.

\section{Interactions in the modality effect}

In separate GLM repeated measures analyses, preferred mode of presentation did not interact with time and condition for retention, $F<1$, or transfer, $F<1$. Spatial ability and prior knowledge did not interact with time and condition for retention, $F<1$, or for transfer, $F<1$.

\section{Discussion}

The goal of the present study was to study the modality effect in children more deeply, taking different learning and cognitive characteristics into consideration, and looking at long-term effects. Our first hypothesis was that children who score high on learning and cognitive measures would score higher overall on the retention and transfer questions. Correlation analysis indeed showed this general effect for reading comprehension, general school performance, prior knowledge, and auditory and visual working memory, which is in line with the Matthew effect (Stanovich 1986). It is unclear why these effects were not found for the other cognitive and learner characteristics. One explanation is that the test was not hard enough for children to differentiate them on the basis of differences on spatial ability and technical reading. Learning eagerness did not have an effect because the majority of the children chose the same answer, namely option 3 on a five-point continuum.

The second hypothesis expected a modality effect immediately after the intervention for retention but not at the second and third testing occasions, thereby replicating and extending the results found by Segers et al. (2008). The second part of this hypothesis could be confirmed; there were no differences between the reading and listening condition at T2 and T3. However, at T1, we found a reversed modality effect. This is contrary to 
the findings reported in the literature with adults, often using a system-paced design. It is in line, however, with studies that used a learner-paced design. (e.g. Tabbers et al. 2004). Segers et al. also found a reversed modality effect but also a normal modality effect immediately after the intervention. A difference between the present study and the study by Segers et al. (2008) could perhaps explain the results: Segers et al. tested retention effects by having children answer questions which on the short term had an advantage for the oral condition. In our study, the children had to write down what they remembered. This requires a more active way of remembering because recall is much less prompted in this type of testing. Another explanation could be is that reading is a more active process than listening because reading activates both orthography and phonology (Nelson et al. 2005), while listening does not. The memory trace of reading includes both orthographic and phonological information, while listening does not. This explanation is not completely satisfying because the effect does not remain over time. Why this is the case needs to be explained by additional research.

Our third hypothesis was to find the modality effect for transfer immediately after the intervention and a reversed modality effect at the second and third measurement. This hypothesis can be confirmed in part. We did find a reversed modality effect at the second testing occasion. It is striking to find that the children in the reading condition have a higher score at T2 than at T1 (such as in Segers et al. 2008). The higher scores in the reading condition at $\mathrm{T} 1$ in retention resulted into higher transfer effects after one night. The fact that the results disappeared at $\mathrm{T} 3$ can have various explanations. The children were tested for the third time at that point, and a loss of interest in the subject could have influenced the results. Again, we did not find the expected modality effect at $\mathrm{T} 1$ and therefore, did not replicate the results from the previous studies with adults.

Regarding the fourth hypothesis, we found no interaction effects between learning and cognitive measures and time or condition. As indicated by Ginns (2005), modality effects are less strong if the learning process is user-paced. This was the case in our study, and perhaps this suppressed the effects of prior knowledge, spatial ability and preferred mode of presentation on the modality effect. One variable that showed a main effect in all of the analyses was reading comprehension. This was an important predictor of success in learning, both in the visual and the auditory condition. Reading comprehension also correlated with other learner and cognitive characteristics (e.g. general school performance) and thus, seems to be a very general measure of school ability.

A limitation of the present study was that all of the children were required to type in their answers in the computer no matter what their preferred mode of presentation or what the condition the children was in. It could be the case that children with high auditory skills only score better if the subsequent tests are administered orally (Rickheit et al. 1987; Low \& Sweller 2005). It would also be interesting to see if the form of testing enhances the modality effect found with adults. This type of research also simulates a more ecologically valid situation. Although the majority of the tests are still administered in a written fashion, there is an increase in oral tests in today's educational climate in order to better suit the need of children with learning disabilities such as dyslexia.

In this design, it was not possible to control whether children had studied all the information in the intervention. A global view of the behaviour of the children could be gathered by logging the activity on the computer screen or using an eye tracker. In addition to checking whether participants study all the information (especially in the visual condition), the eye tracker allows researchers to see how long participants study the pictures when the corresponding information is presented visually or auditorily. If all the information is presented visually, participants have the possibility to focus on one type of information (text or picture) first or switch between the two regularly. People in the auditory condition, however, cannot switch between the two types of information. Research has indicated that when narration was added, (adult) participants spent more time fixating on pictures than when narration was absent (Slykhuis et al. 2005). It is not clear, however, if this effect also holds for children. When designing a cross-sectional eye-tracking study with adults and children from different ages, it is possible to see whether children learn differently than adults. This information can shed more light on the difference in results found in the present study and studies that did find a modality effect.

To conclude, the present study did not find any evidence for a modality effect in children when the lesson 
is learner-paced. Instead, we found a reversed modality effect directly after the intervention for retention and a similar effect for transfer after one day, even after controlling for individual differences. Taking into account the practical implications of this study, the present research sees an advantage of text when this information is accompanied by representational pictures. As the computer becomes more prevalent in today's educational system, children will encounter more and more multimedia learning environments. The advantage of this development for children, however, is not clearly demonstrated from this study. Implementing multimedia lessons in primary school is therefore not necessarily a change for the better.

\section{Acknowledgements}

The authors would like to thank the anonymous reviewer for his or her comments that made a significant impact in the improvement of this paper. Also thanks to Liesbeth Linssen for her help in conducting the analyses.

\section{References}

Baddeley A.D. (2000) The episodic buffer: a new component of working memory? Trends in Cognitive Science 4, 417423.

Baddeley A.D., Gathercole S.E. \& Papagno C. (1998) The phonological loop as a language learning device. Psychological Review 105, 158-173.

van Boxtel H.W., Snijders J.Th. \& Welten V.J. (1980) Voorlopige verantwoording en handleiding [Temporary Account and Manual]. Vorm III ISI-Reeks WoltersNoordhoff, Groningen/Swets \& Zeitlinger, Lisse.

Brunken R., Plass J.L. \& Leutner D. (2004) Assessment of cognitive load in multimedia learning with dual-task methodology: auditory load and modality effects. Instructional Science 32, 115-132.

Dean R.S., Yekovich F.R. \& Gray J.W. (1988) The effect of modality on long-term recognition memory. Contemporary Educational Psychology 13, 102-115.

Ginns P. (2005) Meta-analysis of the modality effect. Learning and Instruction 15, 313-331.

van Hoorn W., van der Kamp L. \& den Brinker W. (2003) Nederlandse Differentiatie Testserie NDT-2003. Swets \& Zeitlinger, Lisse.

Jeung H.J., Chandler P. \& Sweller J. (1997) The role of visual indicators in dual sensory mode instruction. Educational Psychology 17, 329-343.
Kozhevnikov M., Hegarty M. \& Mayer R.E. (2002) Revising the visualizer-verbalizer dimension: evidence for two types of visualizers. Cognition and Instruction 20, 47-77.

Low R. \& Sweller J. (2005) The modality principle in multimedia learning. In The Cambridge Handbook of Multimedia Learning (ed. R.E. Mayer), pp. 147-158. Cambridge University Press, New York.

van der Lubbe M. (2008) Terugblik en resultaten Eindtoets Basisonderwijs 2008. Cito, Arnhem.

Mann B., Newhouse P., Pagram J., Campbell A. \& Schulz H. (2002) A comparison of temporal speech \& text cueing in educational multimedia. Journal of Computer Assisted Learning 18, 296-308.

Mann B.L. (2008) The evolution of multimedia sound. Computers \& Education 50, 1157-1173.

Mayer R.E. (1997) Multimedia learning: are we asking the right questions? Educational Psychologist 32, 1-19.

Mayer R.E. (2001) Multimedia Learning. Cambridge University Press, Cambridge.

Mayer R.E. (2003) The promise of multimedia learning: using the same instructional design methods across different media. Learning and Instruction 13, 125-139.

Mayer R.E. \& Massa L.J. (2003) Three facets of visual \& verbal learners: cognitive ability, cognitive style, and learning preference. Journal of Educational Psychology 95, 833-846.

Mayer R.E. \& Moreno R. (1998) A split-attention effect in multimedia learning: evidence for dual processing systems in working memory. Journal of Educational Psychology 90, 312-320.

Mayer R.E., Heiser J. \& Lonn S. (2001) Cognitive constraints on multimedia learning: when presenting more material results in less understanding. Journal of Educational Psychology 93, 187-198.

McLelland J.L., McNaughton B.L. \& O’Reilly R.C. (1995) Why there are complementary learning systems in the hippocampus and neocortex: insights from the successes and failures of connectionist models of learning and memory. Psychological Review 102, 419-457.

Mousavi S., Low R. \& Sweller J. (1995) Reducing cognitive load by mixing auditory and visual presentation modes. Journal of Educational Psychology 87, 319-334.

Nelson J.R., Balass M. \& Perfetti C.A. (2005) Differences between written and spoken input in learning new words. Written Language \& Literacy 8, 25-44.

Paas F., Renkl A. \& Sweller J. (2004) Cognitive load theory and instructional implications of the interactions between information structures and cognitive architecture. Instructional Science 32, 1-8.

Paivio A. (1986) Mental Representations: A Dual Coding Approach. Oxford University Press, Oxford. 
Penney C.G. (1975) Modality effects in short-term verbal memory. Psychological Bulletin 82, 68-84.

Rickheit G., Strohner H., Musseler J. \& Nattkemper D. (1987) Recalling oral and written discourse. Journal of Educational Psychology 97, 438-444.

Segers E., Verhoeven L. \& Hulstein-Hendrikse N. (2008) Cognitive processes in children's multimedia text learning. Applied Cognitive Psychology 22, 375-387.

Slykhuis D.A., Wiebe E.N. \& Annetta L.A. (2005) Eyetracking students' attention to PowerPoint photographs in a science education setting. Journal of Science Education and Technology 14, 509-520.
Stanovich K.E. (1986) Matthew effects in reading: some consequences of individual differences in the acquisition of literacy. Reading Research Quarterly 21, 360-406.

Tabbers H.K., Martens R.L. \& Van Merrienboer J.J.G. (2004) Multimedia instructions and cognitive load theory: effects of modality and cueing. British Journal of Educational Psychology 74, 71-81.

Verhoeven L.T.W. (1995) Drie-Minuten-Toets [Three Minute Test]. Cito, Arnhem.

Wechsler D. (1992) Wechsler Intelligence Scale for Children, Vol. 3. Psychological Corporation, London. 